\title{
Dissecting aneurysm diagnosed in general practice
}

Sonia Baban, MD

Bjarte Sørensen, BSC(Med) MB BS, Specialist

in general practice (Norway), FRACGP

Hjelmeland General Practice Surgery

\section{Introduction}

The aorta lends itself to sonography through its position and composition. Population screening for abdominal aortic aneurysms (AAA) by general practitioners (GPs) has been shown to be feasible [1-4]. Also on clinical indication, point-of-care ultrasound (PoCUS) may be an accurate diagnostic tool for aortic pathology in the hands of generalists such as GPs [5] and emergency physicians [6] in the unselected population.

\section{Case report}

We present a case of the discovery of an aortic dissection in a 71-year-old female in our GP clinic in rural southwest Norway. She has a history of hypertension and polycythemia rubra vera. She experienced symptoms of chest pains radiating to the neck and upper extremities the evening before. She sought help with the emergency services that evening, but the pains subsided, an ECG was taken and was concluded to be a normal reading. The pain returned and she was unable to sleep due to intense pains in which the patient felt the sudden need to abduct her upper limbs. Additionally, she experienced paresthaesia in her left arm and intermittent left sided chest pain, along with abdominal and back pain.

The pain kept changing character and she presented with mild to moderate pain in her lower right quadrant that radiated towards her upper right quadrant the next morning, with no dyspnoea or chest pains.

On examination, her inter-arm blood pressure measured a difference of $7 \mathrm{mmHg}$

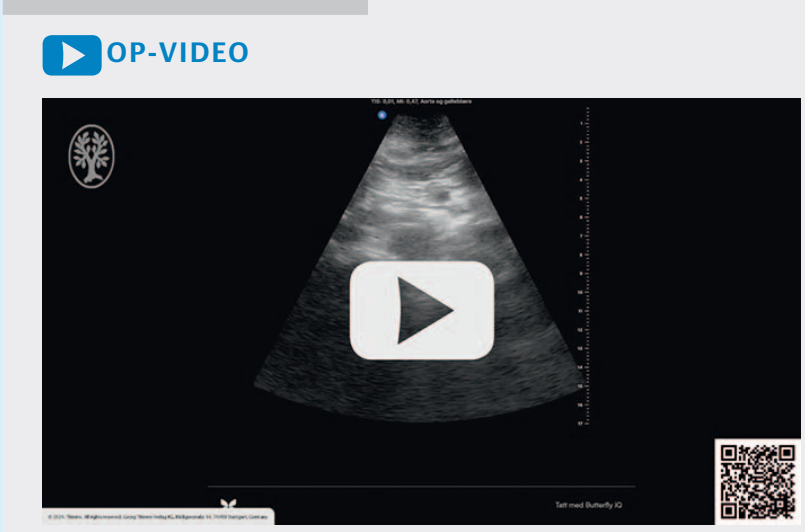

- Video Transverse scanning of the abdominal aorta using a handheld ultrasound device, starting just proximal for the bifurcation and moving proximally towards the epigastric area, interrupted by bowel gas artefacts.

(left arm 162/109 and right arm 155/98) and she had a pulse of 102 . There was general, mild tenderness upon palpation of her abdomen. Her ECG showed a new case of right bundle branch block.

The history and findings suggested an increased pre-test probability for aortic pathology. The patient's abdominal aorta was examined using a handheld portable ultrasound device which showed a pulsating intraluminal structure in the abdominal aorta ( $\triangleright$ Fig. 1). This was confirmed using the practice's stationary ultrasound machine which showed the same pulsating intraluminal structure throughout the whole length of the abdominal aorta, thereby excluding the possibility of an artefact. At this point the patient mentioned that her father died due to an aortic dissection at the age of 70 .

The proximal end of the abdominal aorta measured $3.8 \mathrm{~cm}$ in diameter compared to $2.2 \mathrm{~cm}$ in diameter proximal to the point of bifurcation. A radiologist was consulted by video and the findings of an aortic dissec-

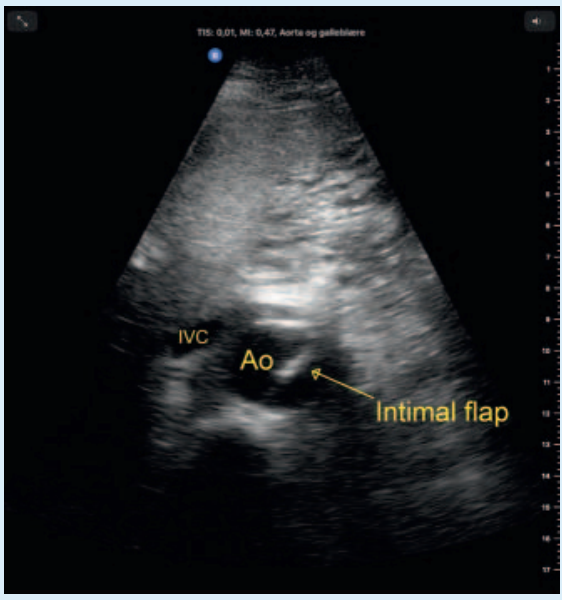

- Fig. 1 Transverse aorta (Ao) mid-abdomen showing intimal flap. IVC = Inferior vena cava.

The patient was air lifted to the hospital where a CT of the aorta confirmed a Stanford type A (De Bakey type I) aortic dissection. She was immediately transferred to the nearest appropriate centre for surgical treatment. 


\section{Discussion}

Although the annual incidence of aortic dissection is as low 2-15 per 100000 [7], and indeed, this being the first case presenting to our rural practice the last decade or so, we were able to accurately diagnose it using ultrasound. The pathognomic pulsating intimal flap was immediately obvious to both authors and is an example of how general practice works in general - having a broad experience in normality and thus a high sensitivity for detecting the abnormal.

The dangers of using PoCUS by generalists in an unselected population with low disease prevalence are of course both overand under-diagnosis. However, with the right level of pre-test probability gained through history taking and examination, research has shown that PoCUS may be a valuable tool in the generalist's hands for a large variety of indications [8]. In our case, we had enough information to admit the patient to hospital regardless of the ultra- sound findings, but they provided reason for immediate large bore vascular access, a much more pointed referral and the choice of a more rapid mode of transfer through helicopter retrieval which would not have been indicated without the diagnosis.

The patient has consented to the case presentation. The authors have no conflict of interests.

\section{References}

[1] Blois B. Office-based ultrasound screening for abdominal aortic aneurysm. Can Fam Physician 2012; 58: e172-e178

[2] Bailey RP, Ault M, Greengold NL et al. Ultrasonography performed by primary care residents for abdominal aortic aneurysm screening. J Gen Intern Med 2001; 16: 845-849

[3] Bravo-Merino L, González-Lozano N, MarotoSalmón R et al. [Validity of the abdominal ecography in primary care for detection of aorta abdominal aneurism in male between 65 and 75 years]. Aten Primaria 2019; 51: 11-17
[4] Sisó-Almirall A, Kostov B, Navarro González M et al. Abdominal aortic aneurysm screening program using hand-held ultrasound in primary healthcare. PLoS One 2017; 12: e0176877

[5] Lindgaard K, Riisgaard L. Validation of ultrasound examinations performed by general practitioners. Scand J Prim Health Care 2017; 35: $256-261$

[6] Rubano E, Mehta N, Caputo W et al. Systematic review: emergency department bedside ultrasonography for diagnosing suspected abdominal aortic aneurysm. Acad Emerg Med 2013; 20: 128-138

[7] Wundram M, Falk V, Eulert-Grehn |] et al. Incidence of acute type A aortic dissection in emergency departments. Sci Rep 2020; 10: 7434

[8] Sorensen B, Hunskaar S. Point-of-care ultrasound in primary care: a systematic review of generalist performed point-of-care ultrasound in unselected populations. Ultrasound J 2019; 11: 31 